\title{
De la lectura y la escritura a la literacidad: Una revisión del estado del arte*
}

\author{
David Alberto Londoño Vásquez ${ }^{* *}$
}

Recibido: 2014-09-17 Aprobado por pares: 2014-10-20

Enviado a pares: 2014-09-28 Aceptado: 2014-11-25

\section{Resumen}

En este artículo se presentan los antecedentes más relevantes frente a la lectura y la escritura, y la aplicación de conceptos como cultura escrita (Emilia Ferreiro), alfabetización académica (Paula Carlino y Giovanni Parodi) y literacidad (Daniel Cassany) en diferentes investigaciones lideradas en algunas instituciones de Educación Superior, haciendo principal énfasis en nuestro país, sin desconocer los aportes teórico-metodológicos e investigativos surgidos tanto en Hispanoamérica como en otros países.

Palabras clave: alfabetización académica, cultura escrita, lectura y escritura, literacidad

Este artículo se desprende de la tesis doctoral Análisis sociolingüístico de los niveles de literacidad en jóvenes de la Institución Universitaria de Envigado, requisito de grado para optar el título de Doctor en Ciencias Sociales, Niñez y Juventud en la Universidad de Manizales-Cinde. La tesis fue dirigida por la Dra. Luz Stella Castañeda Naranjo.

* Docente tiempo completo de la Institución Universitaria de Envigado. Researcher ID: F-8907-2013. ORCID: 0000-0003-1110-7930. Correo electrónico: dalondono@correo.iue.edu.co 


\title{
From Reading to Literacy: A review of the state of the art
}

\begin{abstract}
Relevant background before Reading and Writing and the application of concepts such as written culture (Emilia Ferreiro), academic literacy (Paula Carlino and Giovanni Parodi), and literacy (Daniel Cassany) in different researchs lead by some Higher Education Institutions - with emphasis in our country, bearing also in mind theoretical-methodological and research contributions in both Hispano America and other countries as well - , are presented in this article.
\end{abstract}

Key words: academic literacy, written culture, reading and writing, literacy. 


\section{Introducción}

La lectura y la escritura en los últimos 30 años en Hispanoamérica han venido ganando un espacio significativo en las diferentes discusiones tanto académicas como investigativas de las ciencias sociales y humanas. Al parecer, las diferentes disciplinas y áreas del conocimiento observan en estas un potencial de aprendizaje, generación de conocimiento y diseminación de los procesos y resultados académicos e investigativos, permitiendo que las personas involucradas puedan no solo entender y comprender aquello a lo que acceden, sino también producir y compartir lo aprendido. No obstante, el quid se ha asentado en si los procesos de lectura y escritura deben hacerse desde una perspectiva meramente lingüística o literaria, o si es posible incluir elementos cognitivos o sociocognitivos en el proceso; de igual forma, recientemente, ha aparecido una opción sociocultural.

Cada una de estas opciones teóricas lleva consigo una construcción conceptual que apunta a diferentes rasgos constitutivos y características precisas de lo que sería la comprensión de textos y la producción de los mismos, reconociendo o relegando algunas prácticas. Es por ello que es pertinente la revisión del estado del arte de dichas posturas con el ánimo de poder entender el paso que se ha dado desde la lectura y la escritura a través de la cultura escrita y la alfabetización académicas, hasta llegar a la literacidad'. Este trasegar conceptual se presenta a partir de la división geográfica de sus principales autores y representantes: Antioquia, Colombia, Hispanoamérica y otros lugares, indicando esas diferencias conceptuales.

De igual forma, antes de abordar dicha empresa descriptiva, es oportuno iniciar señalando que la lectura y la escritura han tenido una evolución conceptual muy lenta a través de la historia. En la Antigüedad, leer y escribir no eran consideradas como competencias necesarias para una persona promedio; estas eran solo posibles para los aristócratas. Cabe resaltar que la apertura de instituciones y lugares dedicados al estudio y consolidación de saberes², ha estado presente través de las diferentes épocas; sus intereses académicos

\footnotetext{
La literacidad "abarca todo lo relacionado con el uso del alfabeto: desde la correspondencia entre sonido y letra hasta las capacidades de razonamiento asociadas a la escritura" (Cassany, 2006, p. 38). Esta incluye el código escrito, los géneros discursivos, los roles de autor y lector, las formas de pensamiento, la identidad y el estatus como individuo, colectivo y comunidad, y los valores y las representaciones culturales. Todos ellos, elementos que rigen la interacción de un sujeto crítico con respecto a las diferentes prácticas discursivas, las cuales no solo son el producto del conocimiento (campo disciplinario) sino que tienen un carácter sociocultural, donde el mismo conocimiento se ve afectado por ese contexto. El término es tomado de literacy en inglés cuya traducción es alfabetización al español, pero como se pudo observar, la literacidad dista de la mera alfabetización y sus denotaciones y connotaciones. Vale la pena señalar que el concepto de literacidad se irá ampliando en los diferentes trabajos e investigaciones que se centran en él y fueron retomados en este artículo.

2 Muchas civilizaciones antiguas tuvieron centros de altos estudios. En China se encuentra la más antigua universidad registrada, la Escuela Superior (Shang Hsiang) imperial durante el período Yu (2257 a. C.2208 a. C.). La actual Universidad de Nankín se remonta a la Academia Central Imperial de Nanking, fundada en el año 258. La Universidad de Hunan (Changsha) es continuidad, incluso geográfica, de la Academia Yuelu fundada en el 976.|[ La Universidad de Takshashila, fundada en Taxila (Pakistán) alrededor del siglo VII a. C., entregaba títulos de graduación. La Universidad de Nalanda, fundada en Bihar (India), alrededor del siglo V a. C., también entregaba títulos académicos y organizaba cursos de post-grado.| JEn Grecia, Platón fundó la Academia en el año 387 a. C.
} 
fortalecieron áreas tan específicas como las ciencias exactas, naturales, filosóficas y espirituales. A principios del siglo XVI, se fundaron las primeras universidades en Colombia. En 1592 se creó el Colegio de San Bartolomé, considerado como el más antiguo del país; la Universidad Javeriana en 1623 por los jesuitas; la Universidad Tomasina en 1639 a cargo de los padres dominicos; el Colegio Mayor de Nuestra Señora del Rosario en 1653; y otros colegios en centros urbanos de importancia económica y política, como Tunja, Cartagena, Pamplona, Popayán, Mompox, Antioquia y Honda. El núcleo de la enseñanza de la época fueron las artes, la filosofía y la teología moral, pues la educación que se impartía en estos claustros estaba permeada por el escolasticismo medieval, y tenía como función la legitimación ideológica de la sociedad granadina y sus privilegios de castas ${ }^{3}$.

En otras palabras, los intereses políticos de la época se veían traducidos en los programas académicos ofrecidos, y solo podían acceder aquellos que pertenecían al ilustre mundo de la alta alcurnia colombiana. La tasa de analfabetismo como porcentaje de la población adulta en Colombia (66 \%) se encontraba en 1900 entre las más altas de América Latina. De hecho, Colombia tenía un 20 \% más de personas analfabetas que Argentina. La cifra es más dramática si se compara con Estados Unidos y Canadá. "En 1890 el porcentaje de la población analfabeta mayor de 10 años en los Estados Unidos era de 13.3 \% y el total de la población analfabeta en Canadá en 1861 era 17,5 \%" (Engerman, Mariscal, \& Sokoloff, 2002, p. 16). En 1930, la burguesía liberal proclama la necesidad de una educación popular y se preocupa por acabar con el analfabetismo4. En 1970, Colombia presentaba una tasa de analfabetismo del $22 \%$, cifra superior a la de Argentina que había logrado reducirla a $7 \%$, y Chile y Costa Rica a $11.8 \% .5$ En 1980, Colombia tenía el $15.8 \%$ y al finalizar el siglo XX, el $8.3 \%$. En 2011, el nivel de analfabetismo en nuestro país llega al 6,6\%5.

Es importante señalar que si bien actualmente la tasa de analfabetismo es baja, los niveles de lectura y escritura son preocupantes; es decir, encontramos personas que no comprenden lo que leen y que se les dificulta hacerse entender a través de la escritura, que tienen dificultades de interpretación de significados implícitos y complementarios, que carecen de elementos de análisis y no diferencian entre un argumento y una manipulación.

3 En la segunda mitad del siglo XIX se crean la Universidad Nacional, fundada en 1867 con las facultades de Jurisprudencia, Medicina, Filosofía, Letras, Ingeniería y la Escuela de Ciencias Naturales. Posteriormente se crea la Universidad de Antioquia en Medellín (1871), mediante la fusión del Colegio Máximo del Estado, el Jardín Botánico y la Biblioteca del Estado, y en 1887 se funda la Escuela de Minas de Medellín. Estos tres centros educativos impulsaron la formación de la clase empresarial moderna en la década de 1870. Hacia finales del siglo XIX, además de las universidades del Cauca, de Cartagena, de Nariño, Nacional, de Antioquia, Externado de Colombia -clausurada en 1895 y reabierta en 1918- y el Colegio Mayor del Rosario, la Educación Superior contaba con la Universidad Central, fundada en 1913, y la Universidad Libre, fundada en 1923.

4 Estos altos niveles de analfabetismo a pesar de los esfuerzos de alfabetización que se realizan durante los años cincuenta. En 1958 se crea la Sección de Alfabetización dentro del Ministerio de Educación Nacional y se inicia una campaña basada en la enseñanza directa en escuelas nocturnas.

5 Las estadísticas presentadas fueron tomadas del trabajo La educación primaria y secundaria en Colombia en el siglo XX, realizado por María Teresa Ramírez y Juana Patricia Téllez, en el año 2006, auspiciado por el Banco de la República de Colombia, y del diario El Espectador del 11 de septiembre de 2011 
Por tanto, es relevante revisar y señalar esas investigaciones sobre lectura y escritura realizadas en los niveles local, regional e internacional.

\section{Algunas investigaciones sobre la lectura y la escritura en Antioquia ${ }^{6}$}

Con respecto a Antioquia, autores como Castañeda $E$ Henao (1995, 1999, 2003, 2005), profesores de la Universidad de Antioquia, han realizado diferentes investigaciones relacionadas con la lectura y la escritura, la cultura escrita y, últimamente, la literacidad. En 1995, publicaron el libro La lectura en la universidad. Allí los autores desarrollan la importancia de la lectura, el resumen, lecturas de diferentes textos y, por último, unos talleres de aplicación. Todo esto encaminado a desarrollar las habilidades de lectura y escritura de los estudiantes universitarios, implementando algunos elementos teóricos y metodológicos que plantean criterios para la lectura de textos de diferente tipo, con el objetivo de mejorar la calidad académica de los estudiantes y de los profesionales.

En 1999, publicaron el artículo La lectura en la Universidad de Antioquia: Informe preliminar, donde expusieron una parte de las experiencias que se recogieron a través del curso "Taller de lectura y escritura", que en el segundo semestre de 1998 programó la Facultad de Educación de la Universidad de Antioquia, Medellín, Colombia, para estudiantes de Licenciatura de diferentes áreas. El grupo estaba conformado por 31 estudiantes, algunos eran maestros en ejercicio y los otros se estaban preparando como futuros maestros. Los resultados que se obtuvieron se compararon con los datos que se recogieron en dos cursos similares, uno en la Facultad de Medicina y otro en la de Economía, porque para la realización del proyecto, que empezó a ejecutarse en abril de 1998, el equipo investigador seleccionó tres grupos de estudiantes que recibían el curso de lengua materna en las tres facultades mencionadas y al hacer la evaluación final, el grupo que más avanzó fue el de Educación, hasta el punto que casi obtuvo promedios similares a los de Medicina y Economía; tal vez, porque en Medicina el curso no tenía créditos académicos; es decir, no afectaba su promedio crédito. Sin embargo, al constatar que aún se mantenían algunos problemas de comprensión lectora, los estudiantes solicitaron más cursos de lengua materna.

En 2002, Henao y Castañeda publicaron su libro El papel del lenguaje en la apropiación del conocimiento. Un año después, centran sus intereses investigativos en la lingüística textual $^{7}$ y trabajan alrededor de la cultura escrita, cuyos resultados se ven materializa-

$6 \quad$ Las investigaciones en este apartado se presentan agrupadas según las instituciones y universidades pertenecientes al departamento de Antioquia.

7 La Lingüística Textual hace un principal énfasis en entender el texto como la unidad de análisis, pensando en elementos macroproposicionales, donde cada uno de los elementos que aparecen en la construcción textual cumplen una función. Esta puede ser a nivel micro, macro o superestructural. De igual forma, los elementos de análisis son la cohesión, la coherencia, la concordancia, la adecuación, la informatividad, el registro y los elementos gramáticas del caso, entre otros. Sus implicaciones metodológicas son la deligación de la lingüística tradicional y el desarrollo de habilidades sintácticas, semánticas y pragmáticas. Una explicación más detallada es dada en el artículo Niveles de literacidad de los estudiantes de Psicología de la IUE de Henao, Londoño, Frías y Castañeda (2011), especialmente, en los requerimientos de literacidad para estudiantes universitarios. 
dos en la publicación del artículo: La lingüística textual como alternativa para mejorar la lectura y la escritura en la Educación Superior. Estudio de un caso, y dos años más tarde, los artículos: La lectura y la escritura como problema en el ámbito universitario y la lingüística textual y la cultura escrita en la Universidad. La tesis central de dichos autores propone como base teórica y metodológica para estos cursos, la aplicación de la lingüística textual a los procesos de la cultura escrita. Además, referencian los adelantos de los trabajos teórico-prácticos alrededor de la literacidad realizados por autores como Cassany (2006).

Por otro lado, la tesis doctoral de Mejía (2001) Comprensión y producción del texto escrito. Un enfoque cognitivo-discursivo, cuya población fueron los estudiantes del INEM de Medellín, presenta una propuesta para la utilización de la lingüística textual en la enseñanza del español, teniendo en cuenta que la comprensión y producción del texto es un proceso bidireccional, interactivo y estratégico, que integra los conocimientos previos del lector (cognitivos), los saberes del texto (discursivos) y los elementos del contexto, en búsqueda de la producción de sentido.

Bajo la mirada de la lingüística textual, se destaca el trabajo de investigación de maestría de Maya (2006) denominado La lingüística textual en los procesos de literacia, para el cual tomaron muestras de estudiantes de la Universidad de Antioquia, matriculados en dos grupos diferentes, uno de ellos bajo la propuesta metodológica de la investigadora (grupo experimental) y otro seleccionado al azar (grupo control). Los resultados obtenidos demuestran cómo el empleo de la lingüística textual mejora los niveles de literacidad en los estudiantes universitarios. Fue un buen punto de partida para esta tesis doctoral; sin embargo, dicha investigación no desarrolla ni enuncia los requerimientos que deben tener dichos estudiantes. Tampoco trabaja el concepto de literacidad crítica. Aquí cabe señalarse que esta experiencia fue replicada en la Universidad de Medellín, como se señalará a continuación.

Con respecto a la Universidad de Medellín, se encuentran dos trabajos investigativos que parten de diferentes enfoques: el primero, Lectura y escritura en la Educación Superior realizado por Sánchez y Osorio (2006) donde se centran en el texto escrito como un problema de comunicación surgido específicamente en las aulas de enseñanza de periodismo. Enseñar a redactar un texto informativo breve, la noticia, adquirió una dimensión problémica cuando las técnicas propias del periodismo se quedaron cortas para la fundamentación y análisis del mismo texto y para el diseño de estrategias metacognitivas que contribuyeran a la comprensión de su estructura. Bajo esta mirada, la producción de textos periodísticos debe dinamizarse, debido a los nuevos formatos surgidos durante los últimos 15 años, donde la hipertextualidad permite interactuar en tiempo real. Cabe señalar que esta investigación parte de la propuesta de "entender la información como el proceso mediante el cual alguien que posee un cierto saber lo transmite, con la ayuda de cierto lenguaje, a alguien que, se supone, no lo posee" (Sánchez E Osorio, 2006, p. 12). Esta definición incita a considerar el intercambio de información como una acción de construcción de conocimiento. 
El segundo enfoque aparece materializado, principalmente, en dos investigaciones: La lectura crítica en la Universidad de Medellín ¿Cómo leen y estudian los estudiantes que ingresan al primer semestre?, a cargo de Toro, Arboleda, Henao E Arcila (2008) y La importancia de los cursos introductorios de cultura escrita en ámbitos universitarios, bajo la coordinación de Maya (2008). Ambas investigaciones parten de un enfoque tomado desde la lingüística textual, y los resultados evidencian el mejoramiento en los niveles de literacidad (o cultura escrita), en aquellos estudiantes que estuvieron expuestos a esta experiencia. En términos generales, los estudiantes del grupo control obtuvieron en la prueba final promedios similares a los de la prueba inicial; en cambio, los promedios del grupo experimental fueron significativamente mayores en la prueba final, menos en la variable ortografía.

Bajo otro enfoque teórico, en la Universidad Pontificia Bolivariana, los investigadores del grupo Lengua y Cultura han centrado sus intereses en la lectura y la escritura en la educación superior. Recientemente, Montoya, Arbeláez y Álvarez publicaron los resultados de dos investigaciones: Una propuesta de lectura y escritura en la Educación Superior (2009) y Lectura y escritura en la universidad (2010). En dichos trabajos se señala cómo se pueden mejorar los procesos de escritura en la universidad, a través del desarrollo de habilidades prácticas, las cuales están contempladas en el Proyecto Institucional bajo la denominación de competencias comunicativas. Siguiendo los propósitos de formación del curso, cada docente debe asumir el compromiso de ejercitarse en dichas tareas académicas, para obtener como resultado textos sistemáticos y propositivos, acordes con cada uno de los tipos de escritura y los niveles de lectura que requiere el estudiante en el proceso formativo. Esto permitiría: 1) motivar a la lectura del código escrito y demás sistemas de significación, 2) suscitar el acercamiento a la literatura para el ocio creativo, 3) promover la escritura entre los estudiantes, profesores, empleados y egresados, 4) favorecer el conocimiento de la lengua como vínculo entre el pensamiento y la comunicación efectiva, desde los niveles gramatical, morfológico y semántico, 5) fomentar la oralidad como narración, diálogo, disertación, transmisión de conocimiento.

En lo concerniente a la Universidad EAFIT, a través del grupo de investigación Política y Lenguaje, se lleva a cabo una propuesta con base en la producción y comprensión de textos desde la perspectiva del lenguaje total (López, 2008), en donde señalan las necesidades específicas de desarrollar competencias orales y escriturales por parte de los estudiantes universitarios, vinculando la producción y comprensión textual como resultado de habilidades que incluyen la escucha, el habla y la escritura. Además, el grupo de investigación viene trabajando durante los últimos años, sobre la relación entre las nuevas tecnologías y la relación comunicación y política.

Finalmente, la Institución Universitaria de Envigado (IUE) se suma a la lista de instituciones antioqueñas que han patrocinado investigaciones sobre la lectura y la escritura, si bien es cierto que esta institución solo se ha venido vinculando a estos intereses investigativos desde el año 2010 con el trabajo Cómo leen y escriben los estudiantes 
que ingresan a la Facultad de Ciencias Sociales de la IUE, bajo la dirección de Henao \& Londoño (2010). En esta ocasión, se trabajó con los grupos del curso Taller de Lectura y Escritura I del programa de Psicología de la Facultad de Ciencias Sociales. Se tomó un grupo experimental y se conformó de los otros grupos un número igual de participantes de forma aleatoria como grupo control. A ambos grupos se les realizó una prueba de entrada alrededor del resumen y la lectura crítica; los resultados fueron muy similares. No obstante, el grupo experimental fue intervenido con una propuesta construida desde la lingüística textual, el análisis crítico del discurso y la argumentación pragmadialéctica. Al finalizar el semestre, a ambos grupos se les aplicó una prueba de salida, semejante a la prueba de entrada. Los resultados obtenidos fueron relevantes, puesto que confirman la mejoría de los niveles de literacidad de los estudiantes que participaron en el grupo experimental en comparación con los otros participantes del grupo control, quienes salieron como entraron y que fueron orientados bajo modalidades más tradicionales o con requerimientos teóricos no aplicados a la práctica relacionados con la lingüística textual. Estos resultados motivaron, de igual forma, la realización de la tesis doctoral Análisis sociolingüístico de los niveles de literacidad en jóvenes de la IUE. Este análisis sociolingüístico, el cual no se ha hecho hasta el momento en ninguna de las investigaciones revisadas y referenciadas, permitió correlacionar las variables sociolingüísticas y los niveles de literacidad. A partir de ello, Londoño (2013, p. 79):

Los jóvenes de la Institución Universitaria de Envigado demandan unos niveles de literacidad que potencien una serie de habilidades en relación con la comprensión lectora y la producción escrita de diversos géneros discursivos realizada a través de un proceso que tenga en cuenta los contextos familiares, culturales, disciplinarios, académicos, sociales e individuales.

En otras palabras, dicho proceso no debe ser ajeno a las identidades, las identificaciones, las subjetividades y las ideologías tanto del autor del texto como del lector bajo un enfoque sociocultural, porque la persona que lee y escribe es portadora de una cultura y una visión del mundo; es decir, es un ser sociocultural. Cabe mencionarse que de esta forma, la literacidad no solo es una opción terminológica, sino que también se diferencia de las anteriores en la forma de dinamizar los procesos tanto internos como externos para lograrlo.

\section{Algunas investigaciones sobre lectura y escritura en la educación colombiana $^{8}$}

La preocupación de las universidades colombianas en el tema de los niveles de lectura y escritura ha venido tomando fuerza, dada las altas tasas de deserción académica y las dificultades de comprensión, descripción y argumentación que presentan los estudiantes

Las investigaciones en este apartado se presentan agrupadas según las instituciones y universidades pertenecientes a Colombia. 
universitarios. Algunos de estos esfuerzos se han materializado en la creación de una red colombiana de instituciones de Educación Superior que promueve el diálogo sobre la lectura y la escritura en la universidad, denominada Redlees. La red actúa como una colectividad de orientación y cooperación en las acciones emprendidas por las instituciones y los educadores para fortalecer estos procesos en la formación superior desde el $2006^{9}$.

Sin embargo, la literacidad en Colombia es un tema de discusión todavía en etapa inicial. Incluso el término se utiliza muy tímidamente, porque es extraño para muchos docentes. Apenas en mayo de 2010, la Universidad Autónoma de Occidente de Cali organizó un primer encuentro sobre lectura y escritura en la educación universitaria, que congregó a no más de 50 personas de diferentes universidades del país. Allí se trataron cuatro temas básicos: nuevas tecnologías para leer y escribir en la universidad; formación de docentes universitarios en lectura y escritura; lectura y escritura para la formación de estudiantes universitarios; y políticas institucionales sobre la lectura y la escritura en la universidad.

Al respecto, es importante reconocer la trascendencia de algunas investigaciones realizadas durante los últimos años alrededor de la lectura y la escritura, la cultura escrita o la literacidad, como:

Enseñar a comprender textos en la universidad (Rincón, Narváez y Roldán, 2005), cuyos resultados nos invitan a asumir la lectura y la escritura en la universidad como indagación, exploración, discusión, explicación; acciones propias de la investigación, entendida como una actividad social conducente a la producción de conocimiento. De igual forma, la profesora Rincón, en compañía de un amplio grupo de investigadores de diferentes universidades del país, lideró la investigación ¿Para qué se lee y escribe en la universidad colombiana? (Rincón et al., 2010), donde indagan sobre cuáles son las prácticas de lectura y escritura de las 17 universidades participantes y cómo se caracterizan dichas prácticas. En el informe, Rincón y Gil (2010) señalan que, a partir de una investigación descriptiva e interpretativa basada en información y datos de naturaleza cuantitativa, en las universidades se promueven unos determinados modos de leer y escribir que cumplen con requisitos académicos disciplinares y no necesariamente están enfocados al desarrollo de prácticas de corte investigativo y en pro de la divulgación y formación universitaria.

Bajo un enfoque cercano, el trabajo de Tejada y Vargas (2007) Hacia una integración de la literacidad, la literacidad funcional y la literacidad cultural, propone una puesta en común de las literaturas funcional y cultural con la literacidad, es decir, facilitarles a los participantes la construcción de competencias en literacidad. Para ello, propone la consecución de cuatro fases: descriptiva, interpretativa personal, análisis crítico y acción creativa. De igual forma, argumentan que el énfasis en el desarrollo de habilidades y destrezas (literacidad funcional), tanto para la lectura como para la escritura es importante,

\footnotetext{
9 Aunque, es necesario señalar que desde finales del siglo XX, se realizaron investigaciones centradas en la lectura y escritura a nivel universitario como Castañeda y Henao (1995, 2003, 2005), Cuervo y Flórez (1999, 2005), Moreno (2000), Mejía (2001), Rey (2001), Martínez (2004, 2005), Penagos y Ardila (2004), Sánchez Lozano (2004), Barletta y Mizuno (2005), Cisneros (2005) y Rincón, Narváez y Roldán (2005). No obstante, es a partir de 2006, que surge una red de investigadores alrededor de dicha temática.
} 
pero estas prácticas pedagógicas no se pueden considerar como un fenómeno autónomo, ni neutro desde el punto de vista sociocultural. Además, señalan que hay una relación directa con la literacidad crítica y la democracia, la cual permitiría mejorar las condiciones de la sociedad en general, dada la necesidad de personas autónomas y críticas en pro de la democracia.

Cabe mencionar que la Universidad del Valle cuenta con otros enfoques como los presentados por Ulloa y Carvajal (2005) en la investigación Jóvenes universitarios, cultura escrita y tecnocultura: Análisis de los resultados arrojados por la encuesta en una investigación sobre lectura, escritura, conocimiento y tecnocultura en la universidad, cuyas principales conclusiones apuntan a evidenciar la crisis de la cultura escrita, no solo por la pérdida de importancia en los espacios curriculares y extracurriculares asociados con la lectura y la escritura en los colegios secundarios, sino también por las prácticas juveniles vinculadas a los medios de comunicación y TIC, donde predominan formas de comunicación audiovisual sobre la expresión escrita.

De igual forma, María Cristina Martínez, investigadora de UniValle y directora de la Cátedra Unesco para la Lectura y la Escritura, hace aportes al análisis del texto con trabajos como El procesamiento multinivel del texto escrito (2004) y La construcción del proceso argumentativo en el discurso. Perspectivas teóricas y trabajos prácticos (2005). En su trabajo, presenta el género discursivo como contrato social y centra su atención en la situación de enunciación. De igual forma, diseña una serie de matrices que permiten revisar las relaciones microestructurales, macroestructurales y superestructurales presentes en el texto. Su trabajo pretende conocer acerca de las dificultades que tienen los estudiantes de diversos niveles de escolaridad para comprender y producir textos con organización expositiva, explicativa y argumentativa en diferentes niveles de escolaridad: Básica, Media y Universidad; además, la investigadora impulsa un modelo de intervención pedagógica desde la perspectiva discursiva e interactiva del lenguaje y del aprendizaje, respectivamente, con el fin de incidir en el mejoramiento del rendimiento académico de los estudiantes, desarrollar estrategias que permitan al estudiante aprender a partir de los textos que leen; mejorar el proceso de inferencias textuales en los textos académicos escritos, y avanzar en el desarrollo teórico y metodológico de la lingüística del texto y del análisis del discurso para mejorar el trabajo sobre los niveles de construcción del texto escrito y las posibilidades de transferencia hacia la aplicación pedagógica.

Por otro lado, Lectura y escritura en la universidad: una investigación diagnóstica (Cisneros, 2005) nos ofrece un panorama sobre algunos aspectos en relación con las habilidades de lectura y escritura de los estudiantes que ingresan a la Educación Superior, específicamente a la Universidad Tecnológica de Pereira (UTP). A partir de la lectura de un texto corto y sencillo, de carácter expositivo y argumentativo, que fue aplicado a 1413 estudiantes de diferentes carreras de la UTP, se determinaron las estrategias que usan en el momento crucial de ingreso a la universidad para la resolución de preguntas abiertas que exigen procesos inferenciales; es decir, se trabajaron los procesos de comprensión y de construcción de textos. 
En Barranquilla, Barletta E Mizuno (2005) proponen una metodología para la lectura e interpretación de la información cotextual en un texto de Ciencias Naturales, ya que en su investigación se evidencia que gran número de textos escolares, en Colombia, así como en otras partes del mundo, muestran una serie de rasgos discursivos que pueden dificultar su comprensión por parte de los estudiantes y, por ende, llevan a niveles de aprendizaje inferiores y poco significativos. Por tanto, sugieren que un trabajo colaborativo entre lingüistas y maestros puede facilitar la creación y aplicación de formas de mediar el texto en el aula que, en cierta medida, suplan las deficiencias del texto y permitan alcanzar niveles superiores de aprendizaje.

Además, el Instituto de Estudios en Educación de la Universidad del Norte y la Cátedra Unesco para la Lectura y la Escritura organizaron el Sexto Congreso Internacional de la Cátedra Unesco para el Mejoramiento de la Calidad y Equidad de la Educación en América Latina con base en la Lectura y la Escritura en el año 2011, con una participación de más de 400 ponentes de 17 países. Es importante resaltar que los objetivos de dicho congreso fueron promover procesos de calidad y equidad de la educación en América Latina mediante la enseñanza y el aprendizaje de la lectura y la escritura, socializar los avances en la investigación y en las experiencias didácticas de la lectura y la escritura en América Latina, reflexionar sobre la enseñanza de la lectura y la escritura en los diferentes niveles educativos, y proporcionar herramientas para el mejoramiento de la formación docente y el desempeño académico de los estudiantes.

En Bogotá, sobresalen los trabajos publicados por las profesoras Cuervo y Flórez (1999, 2005) y Moreno (2000), con sus diferentes investigaciones relacionadas con el lenguaje y la escritura, a partir de su participación en la Maestría en Educación de la Universidad Nacional de Colombia en la línea de Comunicación y Educación. Sin embargo, en ninguno de sus trabajos se observa la inclusión de conceptos como la cultura escrita, alfabetización académica o la literacidad, quedándose en aspectos estructurales del lenguaje, la biología y su relación con el aprendizaje.

Por otro lado, el grupo de investigación Lenguaje, Discurso y Saberes de la Universidad Distrital de Bogotá, bajo la coordinación de Rey (2001) ha venido investigando sobre la comprensión y el análisis de las prácticas docentes o formación de maestros, apoyados en los fundamentos teóricos de la pragmática particularmente, el lenguaje y la actividad discursiva en sus relaciones con el contexto praxeológico ${ }^{10}$. El discurso es asumido como una actividad del lenguaje y es estudiado a partir del objeto empírico del texto. Desde esta perspectiva, se han examinado problemáticas como: a) La cultura identitaria de los maestros que busca situar el maestro como intelectual de la cultura; b) La enseñanza de la lectura y la escritura, que surge de la necesidad de potenciar los procesos de lectura

10 La praxeología es la ciencia que estudia la estructura lógica de la acción humana (praxis), centra su atención en el individuo, en el impulso de quien efectiva e indudablemente, actúa, sacando de este estudio axiomas o principios elementales, sólidos e inmutables, con los cuales entender y accionar el proceso del accionar humano. 
comprensiva y producción de textos en el aula, c) La argumentación del discurso del maestro, que pretende desde la perspectiva de los saberes y el análisis del discurso pensar los procesos de comunicación en el aula y posibilitar la emergencia de múltiples textos.

Con respecto a la Universidad Pedagógica Nacional de Bogotá, los intereses investigativos han girado alrededor de las prácticas pedagógicas y el desarrollo de la comprensión y la producción de textos hipermediales. Allí, Penagos \& Ardila (2004) fueron los responsables de la investigación Las prácticas pedagógicas en la formación de lectores y escritores, donde exploraron las prácticas pedagógicas de formación de lectores y escritores, a partir de las concepciones, las tendencias metodológicas y el sentido que los maestros de diferentes áreas de Educación Básica Primaria atribuían a tales prácticas durante los años 2001 y 2004.

Además, el Grupo de Investigación en Hipermedia, Minificción, Literatura y Lenguaje de la misma universidad llevó a cabo la investigación Ambiente hipermedial para el aprendizaje de la literatura y el desarrollo de la comprensión y producción de texto a partir del minicuento (2004), cuyo objetivo fue incorporar las tecnologías de la información y la comunicación en la creación de un ambiente de aprendizaje para el desarrollo de discurso estético y la didáctica de la literatura que tenga como referente el minicuento.

De igual forma, resaltamos el interés de la Universidad Sergio Arboleda en cada una de sus sedes, en cabeza de los trabajos de Sánchez Lozano (2004), quien, a partir de los resultados obtenidos en una investigación, propone cursos de alfabetización académica cuyos objetivos son los de interpretar textos y hacer deducciones e inferencias a partir de sus contenidos; consultar, recolectar y organizar información, para realizar eficazmente las investigaciones disciplinares propias del ámbito académico; producir textos expositivos (informativos, explicativos), haciendo uso de elementos básicos de coherencia y cohesión; hacer conciencia de la importancia social y académica del uso de la ortografía, y reconocer elementos de la argumentación.

Finalmente, la investigación realizada por Flórez y Gordillo en la Universidad de la Salle: Los niveles de comprensión lectora: hacia una enunciación investigativa y reflexiva para mejorar la comprensión lectora en estudiantes universitarios (2009), propende por la educación integral de los estudiantes que ingresan a dicha universidad con base en su Plan Lector. Esta investigación de corte cualitativo tuvo como objetivo establecer el nivel de comprensión lectora de los estudiantes de la Licenciatura de Lengua Castellana, Inglés y Francés, con el propósito de diseñar un programa de intervención desde la lingüística cognitiva. En esta ocasión, los investigadores utilizaron un grupo control y uno experimental, con el ánimo de comparar los diferentes niveles con que ingresaban los estudiantes y aquel con el que salían después de la intervención. Los resultados señalados son positivos frente a lo esperado inicialmente.

Como vimos anteriormente, las investigaciones en el ámbito de la lectura y la escritura han venido posicionándose en Colombia durante los últimos quince años. Sin embargo, este es el efecto de importantes propuestas teórico-metodológicas realizadas en diferentes 
partes de Hispanoamérica y otros países. A continuación se esbozarán las experiencias más relevantes relacionadas con la literacidad.

\section{Investigaciones más relevantes sobre literacidad en Hispanoamérica y otros países}

\subsection{Hispanoamérica}

Indiscutiblemente, los trabajos e investigaciones realizados por Emilia Ferreiro, Paula Carlino, Daniel Cassany y Giovanni Parodi orientan las diferentes propuestas, no solo colombianas como se describió anteriormente, sino también hispanoamericanas.

Desde 1995 hasta 1998, la Secretaría de Educación Pública -SEP- de México, y el Centro Regional para el fomento del Libro en América Latina y el Caribe -Cerlalcrealizaron un proyecto educativo de alcance internacional orientado a la formación de lectores y escritores en escuelas latinoamericanas: Podemos Leer y Escribir (Hederich, E Charria, 1999), el cual partió del hecho de que todos los países de la región estaban comprometidos con el mejoramiento de la calidad de la educación haciendo énfasis en la lectura, la escritura y las habilidades matemáticas. Por ello se propuso que los diferentes países dedicaran enormes esfuerzos a la capacitación de maestros y a la dotación de bibliotecas (de texto, de apoyo curricular y recreativo). No obstante, los resultados de estas acciones no fueron siempre claros, por la ausencia de seguimiento y evaluación sistemática del proceso. En este contexto, el proyecto Podemos Leer y Escribir apoyó la tarea de los diferentes gobiernos en el mejoramiento de la calidad de la formación de niños lectores y escritores en las escuelas de Educación Básica, promovió la evaluación de las acciones en el campo y ofreció herramientas y recomendaciones para la gestión de políticas educativas para desarrollar la cultura escrita ${ }^{11}$.

En Argentina, el trabajo de Carlino (2002) titulado Enseñar a escribir en la universidad: como lo hacen en Estados Unidos y por qué parte de un corpus de análisis compuesto por los documentos elaborados por unas 20 instituciones educativas estadounidenses y la información recogida durante una visita a la Universidad de Princeton. En ellos, según Carlino (2002, p. 59): "Es posible apreciar un panorama distinto al argentino acerca de qué hacen estas universidades con la lectura y escritura de los estudiantes". En los Estados Unidos, cada universidad cuenta con un Programa de Escritura que desarrolla múltiples

1 De acuerdo con los datos disponibles, la cobertura actual del proyecto es verdaderamente significativa. En total, en las 441 instituciones sobre las cuales tenemos información, pueden distinguirse 159796 alumnos, 6184 maestros, 1108 directivos docentes, y 11641 adultos no maestros, directamente participantes en la experiencia. En promedio, cada institución vincula con el proyecto a poco más de 341 alumnos, 14 maestros, y más de 2 directivos (entre los que se cuentan rectores y coordinadores académicos). Calculada la proporción de la institución que directamente participa en esta experiencia, los resultados indican que casi el $80 \%$ de los maestros y el $86.6 \%$ de los alumnos están participando activamente en el programa. Estas cifras muestran una proporción muy alta de la población escolar que se encuentra vinculada con la experiencia El valor del 100 \% de esta relación, corresponde a un proyecto generalizado que se hace institucional, con mayores probabilidades de que pueda pervivir e irse incorporando en el proyecto pedagógico fundamental de la institución. 
intervenciones. Tiene a su cargo una o más materias obligatorias que enseñan escritura académica y diversos cursos electivos de escritura en las disciplinas, para distintos niveles y con diferentes orientaciones, temáticas y perspectivas. Estos programas han organizado Centros de Escritura y ofrecen tutores para discutir los borradores escritos por los estudiantes, quienes llevan sus producciones incipientes, y están dispuestos a reescribirlas antes de entregarlas a los docentes de las materias que se los hubieran encargado. Algunas instituciones también ofrecen asistentes o compañeros de escritura: estudiantes que han sido capacitados y que son asignados a las materias regulares para apoyar a sus profesores a revisar y realimentar los trabajos escritos por los alumnos. Además, es función de estos centros elaborar materiales de orientación para alumnos y docentes. Este enfoque permite que los estudiantes no solo construyan conocimiento a través del proceso de escritura, sino que a través de la práctica, edición y revisión de sus trabajos por una persona con mayor experiencia escritural y perteneciente a su área de formación, vaya consolidando sus conceptos y adquiriendo las competencias escriturales requeridas en el área de formación.

Por otro lado, es relevante reconocer que los aportes de Ferreiro (1997) han sido fundamentales para el desarrollo de nuevas investigaciones alrededor de la cultura escrita y la alfabetización académica, respectivamente. Un ejemplo de ello, es el trabajo de Gallelli y Salles (2000) denominado La investigación-acción en lectura comprensiva. Una propuesta para articular la Educación General Básica (EGB) con el Nivel Medio, cuya etapa inicial fue presentada como ponencia en las Sesiones en Español de la $46^{\circ}$ Convención Anual de la IRA, en la ciudad de Nueva Orleans. Este trabajo permitió la posterior creación de la Red de Lectores de textos literarios y de textos de las ciencias, mediante el empleo del correo electrónico y el correo postal, que permitiese unir a niños, jóvenes y adultos de diversas ciudades del mundo, con el propósito de favorecer las nuevas alfabetizaciones, la inclusión social y la lectura reflexiva de la práctica docente. Dicha red se gestó en agosto de 2004 desde la Dirección del Área de Educación Primaria, organismo dependiente de la Secretaría de Educación (hoy Ministerio) del Gobierno de la Ciudad Autónoma de Buenos Aires.

En Perú, Zavala (2002) lleva a cabo la investigación Negociando prácticas vernáculas: el fracaso de la literacidad en la vida comunal donde analiza los factores sociohistóricos que, en el marco de una ideología de poder y amenaza, influyen sobre la percepción campesina de la literacidad. Desde ahí formula la siguiente pregunta: ¿Qué uso práctico tiene la literacidad para la comunidad campesina y para la vida cotidiana? De igual forma, se resalta el trabajo de Ames (2002), cuyo enfoque etnográfico da luces y propuestas metodológicas diferentes a las planteadas originalmente por la lingüística textual y la literacidad; sin embargo, una no contrapone las otras. Desde otro campo, Revesz (2006) se pregunta qué significa pensar la lectura como un asunto de política pública, lo cual lo motiva a presentar la lectura como un elemento diferenciador en la sociedad y señala su importancia en el mejoramiento de los niveles sociales y económicos. Desarrolla el concepto de literacidad y lo asocia con la cultura escrita. Literacidad y uso social de la lectura y de la escritura. 
El interés hispanoamericano por realizar investigaciones y propuestas concernientes a la cultura escrita, la alfabetización académica o la literacidad se fortalece a través de los años, y esto queda demostrado, especialmente con el trabajo de Londoño E Soler (2004) quienes estuvieron a cargo del texto Significado y alcances de la renovación pedagógica y didáctica de la alfabetización: Plan Iberoamericano de Alfabetización y Educación Básica -PIA- 2005-2015, donde los autores presentan un nuevo concepto de alfabetización con base en la lingüística textual, la literacidad y la cultura escrita (prácticas letradas), las cuales son presentadas como directrices de la Organización de Estados Iberoamericanos (OEI) como plan de mejoramiento.

En España, los trabajos de Camps (1995) Aprender a escribir textos argumentativos: características dialógicas de la argumentación escrita, Camps E Castelló (1996) Las estrategias de enseñanza-aprendizaje de la escritura, Milián $\mathcal{E}$ Castelló (1997) Interacción de contextos en la investigación sobre la composición escrita Castelló (2009), Escribir y comunicarse en contextos científicos y académicos presentan una visión más contextual y flexible para explicar los procesos de composición; es decir, una perspectiva que tuviera en cuenta la importancia de elementos como el contexto social y cultural en el que un texto se inserta, los objetivos personales o las actitudes vinculadas al proceso de escribir como elementos no adyacentes en el proceso de composición de un texto determinado, sino como factores constituyentes y determinantes del proceso cognitivo que el escritor pone en marcha en una situación de comunicación.

En el mismo país, Cassany (2004) dirige el grupo de investigación Literacidad de la Universidad Pompeu Fabra y desarrolla diversas investigaciones alrededor de esta temática, en compañía de importantes investigadores europeos y latinoamericanos. Los temas de interés, inicialmente, estaban relacionados con aspectos generales de la lectura crítica y su fundamentación, los cambios que están creando nuevas prácticas lectoras y el incremento de la importancia de la comprensión crítica. Desde 2006, estos intereses se han tornado hacia la literacidad digital o las formas de lectura en pantalla de los nuevos géneros "tecleados", como el chat, los fórums, el correo electrónico o las webs.

En Chile, además de las numerosas investigaciones de Parodi y su equipo ${ }^{12}$, vale la pena señalar el trabajo de Susperreguy et al. (2007) quienes investigaron Las creencias y prácticas de literacidad en familias chilenas con diferentes niveles educativos. Esta investigación se centró en los niveles de literacidad basado en las creencias y prácticas de los padres chilenos con respecto a sus hijos. Su enfoque fue cuantitativo y se resalta el manejo estadístico, ya que utilizaron una encuesta como instrumento para su posterior análisis factorial de los reactivos del cuestionario con el programa Mplus. La muestra se compone de encuestas realizadas al padre o a la madre de 188 niños matriculados en diferentes instituciones de Chile.

12 Algunas de las investigaciones más relevantes de Parodi son: Conexiones entre lectura y escritura. Aproximaciones discursiva y cognitiva (1999-2002), LECTES, Desarrollo de la competencia estratégica para leer y escribir textos (2002-2005), Ciencias del lenguaje y géneros del discurso (2003-2006), y Discurso académico y discurso profesional: Lingüística de corpus y análisis multidimensional (2006-2009). 
En Venezuela, Serrano (2008) trabaja la alfabetización crítica: conceptualización de las competencias y estrategias de lectura crítica donde propone que la consciencia creciente del valor que tiene la comprensión crítica en el campo de las diversas disciplinas y en el medio social nos permite examinar, desde una perspectiva sociocultural, qué significado adquiere hoy, en la cultura postmoderna, la práctica de la lectura crítica. Por lo tanto, este trabajo: 1) Aborda aspectos teóricos sobre la alfabetización crítica en sus presupuestos básicos. 2) Define y caracteriza la lectura crítica y aspectos conceptuales sobre las competencias que ha de desarrollar el lector crítico y las estrategias de lectura que requiere para abordar con éxito la diversidad de discursos escritos, que le permitan formarse como ser sensible a los valores democráticos de justicia y equidad. Invita a contribuir con la tarea de repensar el currículo en el cual la lectura y la escritura formen parte de la vida de los aprendices, incidan en sus vidas y en los ciudadanos que ellos desean ser.

Nuevamente en México, Ponce E Carrasco (2008) realizaron la investigación Prácticas de lectura en Educación Media Superior. La epistemología personal como recurso de lectura $^{13}$. En dicha trabajo, los investigadores llevan a cabo un estudio comparativo frente a las prácticas de lectura en Noruega, España y México. Con este trabajo se evidencia que la epistemología personal refleja las trayectorias sociodemográficas y lectoras, y condiciona las habilidades de lectura de múltiples textos, según combinaciones entre ingenuidad y sofisticación con respecto a qué es el conocimiento y cómo se construye. Las habilidades de lectura de múltiples textos son un recurso necesario en la práctica académica universitaria.

Un par de años después, Gasca (2010) investigó el Desarrollo de la literacidad en Internet en estudiantes mexicanos de Bachillerato, donde propone que la modificación de las prácticas lectoras en el siglo XXI, debido a una mayor diversidad de textos, formatos y lenguajes nuevos, en contextos distintos, plantea la necesidad de desarrollar la literacidad como parte de una competencia comunicativa más integral. Debido al sentido amplio que implica la literacidad, el presente estudio se centra en el proceso de la búsqueda y análisis de información en Internet y de los documentos seleccionados en torno al tema "violencia en la adolescencia", en un grupo de estudiantes de bachillerato. La investigación, de índole cualitativa, consistió en un estudio de caso instrumental donde se analizaron las estrategias de planeación y búsqueda de información; los criterios que emplean para revisar el rigor, credibilidad y fiabilidad de las páginas web que consultan, así como la calidad de los textos elegidos, y finalmente su interpretación sobre el tema. Los resultados indican que los alumnos muestran una competencia gramatical y semántica, pues descodifican el sistema escrito y construyen significados; sin embargo, no manifiestan la competencia pragmática ni la crítica, ya que no asumen el rol de usuario comunicativo del texto ni de crítico o analista del mismo. Es así que, aunque no desarrollan una literacidad de manera integral, sí logran un avance en la recuperación de información de textos en Internet, donde el apoyo del docente es esencial para guiar y dirigir dicha búsqueda.

13 La epistemología personal se construye a través de las experiencias, vivencias, condiciones, estados, contactos, creencias. 
En el mismo año en Argentina, Moyano (2010) publica su artículo Escritura académica a lo largo de la carrera: un programa institucional, donde comparte su propuesta de enseñanza de la lectura y la escritura basada en el concepto de género en el nivel superior. Esta propuesta propone como objetivo promover el desarrollo de los estudiantes en el uso del lenguaje propio de ámbitos institucionales como la academia, la ciencia y los espacios de actuación profesional. La perspectiva teórica seleccionada es la lingüística sistémico-funcional (Halliday, 1982). Esta es una propuesta para el desarrollo de habilidades de lectura y escritura en la formación universitaria, basada en la teoría de género y registro. La investigadora señala que "El programa, que cuenta con un grado alto de institucionalización, ha sido implementado en el segundo ciclo de las carreras y exige el compromiso de actores de diferente jerarquía institucional y diferentes especialidades disciplinares, además de los docentes en ciencias del lenguaje" (Moyano, 2010, p.465).

Finalmente, cabe resaltar que la importancia de la temática en el ámbito hispanoamericano es tan relevante que en el 2010 se celebraron las III Jornadas Iberoamericanas sobre Prácticas de Lectura y Escritura, y el II Foro Iberoamericano sobre Literacidad y Aprendizaje: "Escribir y leer hoy en la escuela, la biblioteca y otros contextos sociales: prácticas e investigación". Ambos eventos tienen el propósito de abordar prácticas del lenguaje que se organizan en distintos escenarios sociales, principalmente en centros educativos y bibliotecas, como situaciones que propician el desarrollo de la competencia en comunicación lingüística. Estos eventos, organizados por la Asociación Española de Lectura y Escritura (AELE), el Grupo de Investigación Fecomun-Universidad Complutense de Madrid y la Red Ciudades y Pueblos Lectores y Escritores (CPLE) tuvieron como objetivos: Brindar una plataforma interdisciplinar que favorezca la participación de la población infantil y adolescente en distintas prácticas de escritura, lectura y conversación (en centros educativos, bibliotecas y otros ámbitos de la comunidad); analizar las posibilidades que ofrece la literacidad (es decir, prácticas de lectura y escritura acordes con la amplitud y la variedad que nos plantea la sociedad actual), para el desarrollo de la competencia en comunicación lingüística, realimentar las actuaciones de la "Red Ciudades y Pueblos Lectores y Escritores" (Red CPLE) y del Club Telémaco, "Escribir como Lectores", a través del intercambio y el análisis reflexivo de experiencias; potenciar la innovación en las prácticas de lectura y escritura, en distintos contextos escolares y culturales, desde la perspectiva de la inclusión de la diversidad; y establecer puntos de unión entre los profesionales e investigadores que faciliten la generación de proyectos compartidos en torno a las prácticas de escritura y de lectura.

\subsection{Otros países}

Con respecto a investigaciones sobre literacidad realizadas en comunidades académicas por fuera del ámbito latinoamericano tenemos: en Estados Unidos, Behrman (2006) revisó los artículos publicados entre 1999 y 2003 en The Journal of Adolescent and Adult Literacy que estuvieran centrados en lecciones y unidades que enfatizaran la pedagogía de la literacidad en las escuelas y colegios. La metodología de Behrman incluía la búsqueda de 
bases electrónicas de datos utilizando la palabra clave: literacidad. Este solo encontró 36 artículos que presentaran tales características. Finalmente, su investigación reveló que la mayoría de las prácticas comúnmente utilizadas por los docentes en clase son: la lectura de textos suplementarios, la lectura desde una perspectiva de resistencia (oposición al gobierno, al capitalismo, las grandes compañías multinacionales), la producción de textos emancipatorios, la consulta sobre intereses personales de los estudiantes y la sugerencia a tomar acciones sociales.

Por otro lado, en Europa se han realizado un gran número de investigaciones con estudiantes de educación media superior y superior que muestran la relación de las creencias con aspectos como la motivación hacia los estudios, el funcionamiento de los procesos cognitivos, el desarrollo intelectual o el rendimiento académico (Stromso et al., 2006). En 2006, el equipo noruego conformado por Braten, Stromso y Samuelstuen realizó una investigación sobre la forma en la que los estudiantes universitarios y de bachillerato leen múltiples textos, actividad que comúnmente deben realizar, pero para la cual no han sido debidamente preparados en los niveles predecesores. Intentan con este estudio explicar los rasgos que puedan modificar el papel de los elementos involucrados en la lectura. Definen dichos elementos como: el lector, la propia actividad de lectura y el texto. Para el lector, los rasgos a identificar son sus creencias epistemológicas, el interés personal, el conocimiento previo, los recursos de conocimiento y las habilidades de decodificación de palabras. Para la lectura, les interesa observar el uso de estrategias de procesamiento de información; y para el texto, están interesados en emplear múltiples recursos de información (lectura de múltiples textos) frente a la tradicional tendencia de referir la lectura a un solo texto.

La investigadora inglesa Fisher (2008), con base en las propuestas teóricas de Freebody y Luke (1990) y Green (2001), analiza las consecuencias de la implementación de la estrategia nacional de literacidad en las escuelas del Reino Unido. Dicha estrategia les solicita a los profesores de primaria sustituir la práctica de lectura en voz alta, una práctica profundamente arraigada en la pedagogía del Reino Unido, por una lectura guiada, una práctica centrada en la comprensión interpretativa y crítica en lugar de precisión y fluidez. Fisher (2008) sugiere que la enseñanza eficaz de la lectura guiada depende tanto de la comprensión de su soporte psicológico, como de la habilidad del maestro, a través de compartir la responsabilidad para resolver problemas con los niños, para construir puentes entre lo conocido y lo nuevo.

Desde una perspectiva teórica socio-crítica, Jowallah (2007-2009), en sus trabajos sobre la literacidad denominados Critical literacy in England: Utilizing critical literacy pedagogy within the English National Curriculum at Key Stage Three: A case study within a secondary school, compara las diferentes prácticas establecidas oficialmente en los currículos de los colegios ingleses con respecto a la enseñanza de la lectura crítica, lo que refuerza el interés no solo por el desarrollo de competencias lecto-escriturales óptimas sino también la necesidad de identificar las propuestas ideológicas y culturales inmersas en las diferentes prácticas sociales, principalmente la lectura. 
Cabe resaltar que este autor, también ha desarrollado investigaciones comparativas entre los niveles de literacidad en países como EE. UU., China y Jamaica: Using technology supported learning to develop active learning in higher education: A case study (Jowallah, 2008a) y Journeys of Jamaican teachers in England and the United States of America (from 2001 to present), 'Diversity in Organization, Communities and Nations (Jowallah, 2008b). En la primera investigación, se centró en la medición de niveles de aprendizaje activo a través de la utilización de la tecnología en pro del desarrollo de competencias como la literacidad. Con respecto a la otra investigación, resalta el papel de los docentes en la construcción de un pensamiento crítico con base en posturas ideológicas propuestas por organizaciones, comunidades y naciones.

En Australia, Rose en compañía de otros investigadores de la Universidad de Sidney, vienen implementando un programa de lectura y escritura para estudiantes de Primaria, Secundaria y universidad en más de 90 instituciones. Este programa es conocido como Reading to Learn. Rose (2005) en su artículo Learning to Read, el Learning To Read: Reading To Learn: Submission to the National Inquiry into the Teaching of Literacy señala cómo los resultados cuantitativos obtenidos en las últimas mediciones han permitido que los estudiantes con altos niveles de lectura y escritura sigan mejorando sus competencias. De igual forma, pasa con aquellos estudiantes con bajos niveles de lectura y escritura. No obstante, la mejoría de estos últimos es proporcionalmente superior a la de los anteriores. Esto significa que los estudiantes que participan del programa obtienen resultados positivos, los cuales son más visibles en los estudiantes que inicialmente tenían los menores resultados. Además, desde un punto de vista cualitativo, la asistencia a los cursos ha aumentado, y la disposición para las clases es activa.

A partir de las investigaciones anteriores, también nos preguntamos hasta qué punto los jóvenes universitarios han desarrollado los niveles de literacidad, de tal manera que les permita apropiarse de la información que expresan los textos, y así puedan captar lo esencial para rendir con eficiencia en todas las asignaturas y para convertirse en futuros profesionales con plena competencia para desempeñarse adecuadamente en la esfera laboral, y con la capacidad de lectura suficiente para seguir actualizándose por su propia cuenta, lo mismo que para rendir informes escritos en forma coherente y cohesiva; además, con un estilo propio y claro.

Si retomamos ahora el desarrollo de los niveles de literacidad, mirándolos desde la Educación Superior, podemos afirmar que si el estudiante que logra ingresar a la universidad viene de un sistema educativo que no lo ha dotado de las herramientas ni de la formación de hábitos de lectura, que lo habiliten como un lector autónomo, es lógico que en la universidad presente dificultades para la producción y la interpretación de textos escritos tal como lo plantean Castañeda y Henao (1995, 2003, 2005).

Es así como los niveles de literacidad son los ejes fundamentales del trabajo intelectual, porque a través de estas habilidades el estudiante se apropia de los conocimientos y los socializa; entonces, podemos preguntarnos: ¿̇Hasta qué punto estas carencias afectan 
el rendimiento académico del estudiante universitario? Ante esta situación, es necesario que las universidades realicen campañas y brinden asesorías para que en las escuelas y en los colegios se mejore la calidad de la educación a través de la formación de maestros, padres, niños y jóvenes lectores.

\section{Conclusiones}

Como hemos podido observar a través de las diferentes posturas de los trabajos revisados, desde 1990 se han venido presentando distintas propuestas con el fin de mejorar los niveles de lectura y escritura. Cada propuesta es la respuesta histórica a un problema específico.

Al respecto, los trabajos de alfabetización en los niños realizados por Ferreiro (1997) se basan en la necesidad de construir los cimientos de futuros ciudadanos, partiendo de un niño pensante, participante, dinamizador, es decir, un niño que entiende y propone, un niño piagetano. Esta asimilación de un sujeto constructor le permite a la autora centrarse en qué ideas son las que los sujetos expresan en la escritura; es decir, para qué les sirven a ellos la lectura y la escritura. En este caso, es el sujeto quien debe decidir qué leer y para qué hacerlo, facilitando la autonomía. Cabe señalarse que dentro de la propuesta de Ferreiro, se utiliza el término cultura escrita, la cual podría entenderse como la construcción de habilidades y competencias en lectura y escritura para fortalecer los procesos de autonomía y participación, concepción muy cercana a la literacidad, salvo que la forma de alcanzarla es diferente, ya que Ferreiro (1999) se sigue centrando en los procesos de alfabetización.

Desde otra perspectiva, Carlino (2002) sustenta la necesidad de mejorar los niveles de lectura y escritura de los jóvenes universitarios, cuyos niveles son relativamente bajos, dependiendo de la formación previa. En esta perspectiva, el trabajo de preparación, capacitación y consolidación está dado por la disciplina, y es allí donde los docentes de los diferentes cursos deben acompañar el proceso de lectura y escritura, con un propósito más amplio que la alfabetización, la alfabetización académica. Este término invita a pensar la construcción de conocimiento a medida que se adquieren las diferentes formas de leer y escribir en la disciplina de formación. Esta perspectiva lleva a Parodi (2005b) a proponer que es en el lenguaje especializado donde los niveles tanto de lectura y escritura como del conocimiento se amalgaman; en otras palabras, este lenguaje especializado permite entender de forma más precisa y eficiente los textos que se leen, dinamiza los procesos de una escritura coherente y cohesionada, y consolida conceptos, los cuales luego permitirán generar un nuevo conocimiento.

Indiscutiblemente, cada autor y perspectiva presentan elementos y conceptos vitales que han dinamizado las nuevas propuestas de alfabetización; sin embargo, para este proyecto, el término a utilizar es la literacidad, teniendo en cuenta que más que un proceso de alfabetización, el cual es relevante como paso inicial, se da un énfasis en la estructuración y construcción de competencias en lectura y escritura que permiten generar no solo mejores resultados académicos, aunque no dependan exclusivamente del papel de los docentes de las disciplinas específicas, sino también construir lectores 
críticos, autónomos y democráticos, puesto que nuestra propuesta con base en el trabajo de Cassany (2004, 2005c, 2006a) es incluyente y toma de forma coherente y concatenada los conceptos más relevantes de cada una de las propuestas anteriores.

Cabe mencionar que la literacidad no es una sola ni se aprende para siempre; es una serie de competencias que están en constante evolución y construcción, dependientes del contexto y de la situación, que según los niveles, les permitirá ser lectores críticos con competencias discursivas capaces de producir textos con mejor coherencia, cohesión, adecuación, etc., con estructura textual sólida, y no escritos desarticulados, que no diferencian el lector del autor ni dan información nueva sobre el campo del conocimiento en el que se desenvuelve los estudiantes. Si bien la intensidad horaria, y la cantidad de estudiantes por grupo dificultan alcanzar los logros esperados, se avanza en términos significativos en la apropiación de los elementos básicos para una adecuada producción e interpretación textual.

\section{Referencias}

Ames, P. (2002). Para ser iguales, para ser distintos. Educación, escritura y poder en el Perú. Lima: Instituto de Estudios Peruanos.

Barletta, N. E Mizuno, J. (2005). Una propuesta para el manejo del lenguaje del texto de Ciencias naturales. En Zona Próxima, 1 (6), pp. 32-47. Barranquilla: Universidad del Norte.

Behrman, E. (2006). Teaching about language, power, and text: A review of classroom practices that support critical literacy. En Journal of Adolescent and Adult Literacy, 49 (6), pp. 490-98.

Camps, A. (1995). Aprender a escribir textos argumentativos: características dialógicas de la argumentación escrita. En Comunicación, Lenguaje y Educación, 26 (1), pp. 51-64.

Camps, A. E Castelló, M. (1996). Las estrategias de aprendizaje-enseñanza en la escritura. En Monereo, C. E Solé, I. (Coords.). El asesoramiento psicopedagógico y perspectiva profesional y constructivista. Madrid: Alianza, pp. 321-342.

Carlino, P. (2002). Enseñar a escribir en la universidad: Como lo hacen en Estados Unidos y porque. En Uni-pluri/versidad, 2 (2), pp. 57-67.

Cassany, D. (2004). Explorando las necesidades actuales de comprender. Aproximaciones a la comprensión crítica. En Lectura y Vida, 25 (2), pp. 6-23.

Cassany, D. (2006). Tras las líneas. Sobre lectura contemporánea. Barcelona: Anagrama.

Castañeda, L. S. E Henao, J. I. (1995). La lectura en la Universidad. Medellín: Universidad de Antioquia.

Castañeda, L. S. E Henao, J. I. (1999). La lectura en la Universidad de Antioquia: informe preliminar. En Lingüística y Literatura, Medellín, Universidad de Antioquia, pp. 76-85.

Castañeda, L. S. E Henao, J. I. (2002). El papel del lenguaje en la apropiación de conocimiento. Bogotá: ICFES

Castañeda, L. S. E Henao, J. I. (2003). La Lingüística Textual como alternativa para mejorar la lectura y la escritura en la Educación Superior. Estudio de un caso. En Actas Pedagógicas, 2 (1), pp. 76-84. 
Castañeda, L. S. E Henao, J. I. (2005) La lingüística textual y la cultura escrita en la universidad. En Zona Próxima, (6), pp. 12-31. Barranquilla: Universidad del Norte.

Castelló, M. (Coord.) (2009). Escribir y comunicarse en contextos científicos y académicos. Barcelona: Editorial GRAO.

Cisneros, M. (2005). La Lectura y escritura en la universidad: una investigación diagnóstica. Pereira: Universidad Tecnológica del Pereira.

Cuervo, C. E Flórez, R. (1999). Aprender y enseñar a escribir. Bogotá: Programa RED; Universidad Nacional de Colombia.

Cuervo, C. E Flórez, R. (2005). El regalo de la escritura. Bogotá: Unibiblos.

Ferreiro, E. (1997) Alfabetización. Teoría y práctica. México: Siglo XXI Editores.

Ferreiro, E. (1999). Cultura escrita y educación: conversaciones con Emilia Ferreiro. México: Fondo de Cultura Económica.

Fisher, A. (2008). Teaching Comprehension and Critical Literacy: Investigating Guided Reading in Three Primary Classrooms. En Literacy, v. 42 n. ${ }^{\circ} 1$ pp. 19-28.

Flórez, M. P. E Gordillo, A. (2009). Los niveles de comprensión lectora: hacia una enunciación investigativa y reflexiva para mejorar la comprensión lectora en estudiantes universitarios. En Actualidades Pedagógicas, (53), p. 95-10. Bogotá: Universidad La Salle,

Freebody, P. E Luke, A. (1990). Literacy programs: debates and demands in cultural context. En Prospect, (5), pp. 7-16.

Gallelli, G. E Salles, N. (2000). La investigación-acción en lectura comprensiva. Una propuesta para articular la Educación General Básica (EGB) con el Nivel Medio. En: Memorias de la IVL Convención Anual de la IRA, en la ciudad de Nueva Orleans. Disponible en http://aal.idoneos.com/index.php/ Revista/A\%C3\%Blo _ ${ }^{5}$ _ Nro. _ 4/AAL _ en _ EEUU (Consultado: 17/05/2011).

Gasca, M. A. (2010). Desarrollo de la literacidad en Internet en estudiantes mexicanos de Bachillerato. En Memorias del Congreso Internacional Europa/América Latina ATEI Alfabetización mediática y culturas digitales.

Green, P. (2001). Critical Literacy Revisited. En: Fehring, H. E Green, P. (ed.). Critical Literacy: A Collection of Articles from the Australian Literacy Educators' Association. Internacional Reading Association. P. 7-63.

Hederich, C. E Charria, M. E. (1999). Impacto del proyecto "Podemos Leer y Escribir" en el desarrollo de la cultura escrita en las escuelas latinoamericanas. En Folios, segunda época, (10). Bogotá: Universidad Pedagógica Nacional, Departamento de Ciencias Sociales.

Henao, J. I. E Castañeda, L. S. (2002). El parlache. Medellín: Universidad de Antioquia.

Henao, J. I. E Londoño, D. (2010). Análisis del discurso en la formación de lectores críticos, autónomos y democráticos. En El libro total. Colombia: SIC Editores

Henao, J., Londoño, D., Frías, L., \& Castañeda, L. (2011). Literacy levels of Psychology Students at Universidad de Envigado. Zona Próxima, (15), 54-77.

Henao, J. I. E Toro, L. C. (2008). Cultura escrita y educación superior: el caso de la Universidad de Antioquia y la Universidad de Medellín. En: Narváez Cardona, E. \& Cadena Castillo, S. (Comp.). Los desafíos de la lectura y la escritura en la educación superior: caminos posibles. Cali: Universidad Autónoma de Occidente, pp. 53-74. 
Jowallah, R. (2008a). Using technology supported learning to develop active learning in higher education: A case study. US-China Education Review, (12), pp. 46-50.

Jowallah, R. (2008b). Journeys of Jamaican teachers in England and the United States of America (from 2001 to present), Diversity in Organization, Communities and Nations. [Informe de investigación]. Disponible en http://www.wlv.ac.uk/default.aspx?page=13205 (Consultado: 25/05/2011).

Londoño, L. y Soler, M. (2004). Significado y alcances de la renovación pedagógica y didáctica de la alfabetización: Plan Iberoamericano de Alfabetización y Educación Básica -PIA- 2005-2015. Organización de Estados Iberoamericanos. Disponible en http://www.oei.es/alfabetizacion/ Londono-Soler.pdf (Consultado: 01/10/2010).

López, S. (2008). Cara a Cara. El poder en las interacciones comunicativas. Medellín, Fondo Editorial Universidad EAFIT.

Martínez, M. C. (2004). El procesamiento multinivel del texto escrito. ¿Un giro discursivo en los estudios sobre comprensión? Disponible en www.unesco-lectura.univalle.edu.co (Consultado: 11/11/2010).

Martínez, M. C. (2005). La construcción del proceso argumentativo en el discurso. Perspectivas teóricas y trabajos prácticos. En: Cátedra UNESCO Lectura y Escritura: Cali: Editorial Taller de Artes Gráficas de la Facultad de Humanidades de la Universidad del Valle.

Montoya, J.; Arbeláez, O. E Álvarez, A. (2009). Una propuesta de lectura y escritura en la Educación Superior. Medellín: Editorial UPB.

Montoya, J.; Arbeláez, O. E Álvarez, A. (2010). Lectura y escritura en la universidad. Medellín: Editorial UPB.

Moreno, M. (2000). Los lenguajes, los saberes y los aprendizajes. En: Aexmun, (48). Bogotá: Universidad Nacional de Colombia, Facultad de Medicina

Moyano, E. (2010). Escritura académica a lo largo de la carrera: Un programa institucional. En Revista Signos, 43 (74), pp. 465-488. Disponible en http://www.scielo.cl/pdf/signos/v43n74/a04.pdf

Parodi, G. (2000). La evaluación de la producción de textos escritos argumentativos: una alternativa cognitivo/discursiva. Revista Signos, 33 (47), pp.151-166.

Parodi, G. (2003). Relaciones entre lectura y escritura: una perspectiva cognitiva y discursiva. Antecedentes teóricos y resultados empíricos. Valparaíso: Ediciones Universitarias de Valparaíso.

Parodi, G. (2005a). La comprensión del discurso especializado escrito en ámbitos técnico-profesionales: caprendiendo a partir del texto? En Revista Signos, 38 (58), pp. 221-267

Parodi, G. (2005b). Comprensión del discurso escrito. Buenos Aires: EUDEBA.

Parodi, G. (ed.). (2010). Alfabetización académica y profesional en el siglo XXI: Leer y escribir desde las disciplinas. Santiago de Chile: Ariel.

Parodi, G. E Gramajo, A. (2003). Los tipos textuales del corpus técnico-profesional PUCV-2003: una aproximación multiniveles. En Revista Signos, 36 (54), pp. 207-223.

Penagos, R. E Ardila, D. (2004). Las prácticas pedagógicas en la formación de lectores y escritores. Disponible en http://www.cerlalc.org/Escuela/secciones/pagina _ ${ }_{2}$ _ experiencias _ 3. html (Consultado: 23/03/2011).

Ponce, R. E Carrasco, A. (2008). Prácticas de lectura en educación media superior y superior. La epistemología personal como recurso de lectura. Disponible en http://www.scribd.com/doc/35529254/ 
Practicas-de-lectura-en-educacion-media-superior-y-superior-la-epistemologia-personal-comorecurso-de-lectura (Consultado: 15/01/2011).

Ramírez, M. E Tellez, J. (2006). La Educación Primaria y Secundaria en Colombia en el siglo XX. Bogotá: Banco de la República de Colombia.

Revesz, B. (2006). ¿Qué significa pensar la lectura como un asunto de política pública? Organización de Estados Iberoamericanos. Disponible en http://www.cerlalc.org/redplanes/secciones/biblioteca/ agenda _ politicas.pdf (Consultado: 15/01/2011).

Rincón, G. E Gil, J. (2010). Estado de tendencias en las prácticas de lectura y escritura académicas en la universidad del valle. En: ¿Para qué se lee y escribe en la universidad colombiana? [Primer informe de la investigación]. Disponible en http://sintesis.univalle.edu.co/2010/septiembre/lectura. pdf (Consultado: 30/04/2011).

Rincón, G.; Roldán, C. E Narváez, E. (2005). Enseñar a comprender textos en la Universidad: análisis de dos casos. Cali: Artes Gráficas-Facultad de Humanidades

Rose, D. (2005). Learning to Read, Reading to Learn: Submission to the National Inquiry into the Teaching of Literacy 2005, Department of Education, Science and Training. Disponible en http:// www.readingtolearn.com.au/images/pdf/national_inquiry.pdf (Consultado: 6/03/2013)

Sánchez, C. (2004). Comprensión Textual. Bogotá: Universidad Sergio Arboleda.

Sánchez, J. E Osorio, J. J. (2006). Lectura y escritura en la Educación Superior. Medellín: Universidad de Medellín.

Serrano, S. (2008). La alfabetización crítica: Conceptualización de las competencias y estrategias de lectura crítica. En: Letras, 50 (76), pp. 101-130 Centro de Investigaciones lingüísticas y literarias Andrés Bello.

Stromso, H.; Braten, I, E Saluelstuen, M. (2006-2008). Learning in a Knowledge Society: Constructing Meaning from Multiple Information Sources [Propuesta de proyecto]. Noruega: Universidad de Oslo

Susperreguy, M. I.; Strasser, K.; Lissi, M. E Mendive, S. (2007). Las creencias y prácticas de literacidad en familias chilenas con diferentes niveles educativos. En Revista Latinoamericana de Psicología, 39 (2). Bogotá, Universidad Konrad Lorenz

Tejada, H. E Vargas, A. (2007). Hacia una integración de la literacidad, la literacidad funcional y la literacidad cultural en Colombia. En Lenguaje, 35 (1), pp. 197-219.

Toro, L.; Arboleda, L.; Henao, J. I. E Arcila, M. (2008). ¿Cómo leen y escriben los estudiantes que ingresan a la universidad? En Revista Universidad de Medellín, 43 (85), pp. 123-136. Medellín: Universidad de Medellín.

Ulloa, A. E Carvajal, G. (2005): Jóvenes universitarios, cultura escrita y tecnocultura: Análisis de los resultados arrojados por la encuesta en una investigación sobre lectura, escritura, conocimiento y tecnocultura en la universidad. En: Revista Anagramas, Facultad de Comunicación Social, Universidad de Medellín.

Zavala, V. (2002). Negociando prácticas vernáculas: el fracaso de la literacidad en la vida comunal. En: Taller interactivo: prácticas y representaciones de la nación, estado y ciudadanía en el Perú. Lima, Instituto de Estudios Peruanos. 